\title{
Shot noise in magnetic double-barrier tunnel junctions
}

\author{
T. Szczepański, ${ }^{1}$ V. K. Dugaev, ${ }^{1,2}$ J. Barnaś, ${ }^{3,}{ }^{*}$ J. P. Cascales, ${ }^{4}$ and F. G. Aliev ${ }^{4}$ \\ ${ }^{1}$ Department of Physics, Rzeszów University of Technology, Aleja Powstańców Warszawy 6, 35-959 Rzeszów, Poland \\ ${ }^{2}$ Department of Physics and CFIF, Instituto Superior Técnico, TU Lisbon, Avenida Rovisco Pais, 1049-001 Lisbon, Portugal \\ ${ }^{3}$ Institute of Molecular Physics, Polish Academy of Sciences, Smoluchowskiego 17, 60-179 Poznań, Poland \\ ${ }^{4}$ Departamento de Fisica de la Materia Condensada, C-III, Instituto Nicolas Cabrera and Condensed Matter Physics Center (IFIMAC), \\ Universidad Autonoma de Madrid, 28049 Madrid, Spain
}

(Received 11 February 2013; revised manuscript received 16 March 2013; published 9 April 2013)

\begin{abstract}
We calculate shot noise and the corresponding Fano factors in magnetic double-barrier tunnel junctions. Two situations are analyzed: (i) the central metallic layer is nonmagnetic while the external ones are ferromagnetic, and (ii) all of the metallic layers are ferromagnetic. In the latter case, the number of various magnetic configurations of the junctions is larger, which improves the functionality of such devices. The corresponding shot noise and Fano factor are shown to depend on the magnetic configuration of the junctions. The effect of spin relaxation in the central layer is also taken into account. The theoretical results are compared with experimental data on the shot noise in $\mathrm{Fe} / \mathrm{MgO} / \mathrm{Fe} / \mathrm{MgO} / \mathrm{Fe}$ structures.
\end{abstract}

DOI: 10.1103/PhysRevB.87.155406

\section{INTRODUCTION}

Shot noise is still attracting considerable attention due to its importance-not only for the theory and/or experiment, but more importantly for applications. An understanding of the shot noise is crucial for an appropriate description of fluctuating observables, which one usually assumes as constants. Apart from the shot noise, which is a manifestation of the discrete charge of electrons, there are also other fluctuation-induced contributions to the noise, e.g., due to a finite temperature (so-called thermal noise) or due to a strong disorder in the system. It turns out that measurement of the shot noise provides additional information on quantum and Coulomb correlations, which is not accessible in the measurements of average current. ${ }^{1-13}$

The noise measurements are especially important for the physics of mesoscopic structures, where the current fluctuations are expected to be strongly enhanced. ${ }^{1}$ The shot noise in mesoscopic conductors was studied theoretically more than two decades ago in the pioneer works of Lesovik ${ }^{2}$ and Büttiker et al. ${ }^{3,4}$ Various aspects of the shot noise and its manifestation in transport characteristics of normal-metallic, semiconducting, and superconducting structures have been reviewed by Blanter and Büttiker. ${ }^{1}$

In mesoscopic structures which contain ferromagnetic elements, not only charge but also spin fluctuations contribute to the shot noise. ${ }^{5-7}$ This, in turn, may be important for the characterization and functionality of spintronic devices. ${ }^{14,15}$ It has also been shown experimentally that the Fano factor in a single-barrier magnetic tunnel junction depends on magnetic configuration of the junction, and can be remarkably enhanced for the antiparallel alignment of the electrodes' magnetizations. ${ }^{16-20}$ Even more possibilities have been found in double-barrier tunnel junctions with two ferromagnetic electrodes and one ferromagnetic central layer, ${ }^{21,22}$ where one can distinguish four magnetic configurations corresponding to different alignments of the magnetic moments of all three magnetic layers. ${ }^{23}$ It has also been shown that a simple model based on two well-separated spin channels for electronic transport cannot properly describe the experimental observations without taking into account spin-flip transitions. ${ }^{23}$
PACS number(s): 72.25.-b, 73.40.Rw, 85.75.-d

In this paper, we present in more detail a theoretical model of the shot noise in double-barrier magnetic tunnel junctions. Such junctions consist of two tunnel barriers which separate the central layer (in our case, the central layer can be either magnetic or nonmagnetic) from the left and right magnetic electrodes. For simplicity, we assume that magnetic moments of the electrodes and of the central layer are collinear. Some results have already been presented in Ref. 23. Here, we describe the calculations as well as the corresponding results in more detail. We also provide further experimental data to support the theoretical results.

In Sec. II, we consider shot noise in the case when spinrelaxation processes are absent. We consider there separately the situations with nonmagnetic and magnetic central layers. In Sec. III, in turn, we take into account the spin-relaxation processes in the central magnetic layer. Some simple analytical formulas are derived there in the limit of strong spin relaxation. In Sec. IV, we consider tunnel magnetoresistance, while in Sec. V, we present some experimental data on the shot noise in $\mathrm{Fe} / \mathrm{MgO} / \mathrm{Fe} / \mathrm{MgO} / \mathrm{Fe}$ double-barrier tunnel junctions. The summary and final conclusions are presented in Sec. VI.

\section{SHOT NOISE IN THE ABSENCE OF SPIN RELAXATION}

If spin-flip transitions are absent (or can be neglected), the two spin channels can be considered as fully separated. The shot noise corresponding to a single spin channel, $S_{\sigma}$, can be then calculated from the relevant expression for spinless particles, ${ }^{4}$

$$
S_{\sigma}=\frac{R_{1 \sigma}^{2} S_{1 \sigma}+R_{2 \sigma}^{2} S_{2 \sigma}}{R_{\sigma}^{2}},
$$

where $R_{i \sigma}$ is the resistance of the $i$ th junction in the spin$\sigma$ channel (this resistance generally depends on the applied voltage, which, however, is not indicated here explicitly), $R_{\sigma}$ is the total resistance of the spin- $\sigma$ channel, $R_{\sigma}=R_{1 \sigma}+R_{2 \sigma}$, and

$$
S_{i \sigma}=2|e V| \frac{R_{i \sigma}}{R_{\sigma}} \frac{e^{2}}{h} \operatorname{Tr} T_{i \sigma}\left(1-T_{i \sigma}\right) \simeq \frac{2|e V|}{R_{\sigma}} .
$$


Here, $V$ is the external voltage applied to the system and the index $i=1,2$ refers to the two tunnel barriers (two junctions): $i=1$ for the left barrier and $i=2$ for the right barrier, respectively. In Eq. (2), we assumed that the transmission probability is small, $T_{i \sigma} \ll 1$, for tunneling through each of the two barriers. The shot noise $S$ of the double-junction structure includes contributions from both spin channels, $S=S_{\uparrow}+S_{\downarrow}$.

The average value of charge current driven by the voltage $V$ is

$$
I=V \frac{R_{\uparrow}+R_{\downarrow}}{R_{\uparrow} R_{\downarrow}} .
$$

Thus, from Eqs. (1)-(3), one finds the Fano factor in the absence of spin relaxation in the form

$$
F \equiv \frac{S}{2|e I|}=\frac{\left(R_{1 \uparrow}^{2}+R_{2 \uparrow}^{2}\right) R_{\downarrow}^{3}+\left(R_{1 \downarrow}^{2}+R_{2 \downarrow}^{2}\right) R_{\uparrow}^{3}}{R_{\uparrow}^{2} R_{\downarrow}^{2}\left(R_{\uparrow}+R_{\downarrow}\right)} .
$$

In the case of a symmetric structure, $R_{1 \sigma}=R_{2 \sigma}=R_{\sigma} / 2$, from Eq. (4) one finds $F=1 / 2$. In asymmetric situations, however, the Fano factor is different from $F=1 / 2$. Equation (4) can be used to calculate the Fano factor in a particular magnetic configuration of the junction and, therefore, its variation with changing magnetic state of the system, as will be presented below.

\section{A. Nonmagnetic central layer}

Consider first a double junction with a nonmagnetic central layer and two ferromagnetic external electrodes. Let us begin with the parallel $(\mathrm{P})$ configuration of the magnetic moments of the the left (1) and right (2) electrodes. For convenience, we introduce the parameters $\alpha$ and $\beta_{i}$ defined as

$$
\alpha=R_{2 \uparrow} / R_{1 \uparrow}, \quad \beta_{i}=R_{i \downarrow} / R_{i \uparrow} .
$$

The parameter $\alpha$ takes into account asymmetry between the left and right tunnel barriers, while the parameters $\beta_{i}$ describe the spin asymmetry of both barrier resistances. Note that the above definitions hold for the $\mathrm{P}$ configuration.

Taking into account Eq. (5), one can write the Fano factor in the $\mathrm{P}$ configuration as

$$
F_{P}=\frac{\left(1+\alpha^{2}\right)\left(\beta_{1}+\beta_{2} \alpha\right)^{3}+\left(\beta_{1}^{2}+\beta_{2}^{2} \alpha^{2}\right)(1+\alpha)^{3}}{(1+\alpha)^{2}\left(\beta_{1}+\beta_{2} \alpha\right)^{2}\left(1+\alpha+\beta_{1}+\beta_{2} \alpha\right)} .
$$

If we assume that the magnetic electrodes are made of the same material and the spin-filtering properties of both barriers are the same, $\beta_{1}=\beta_{2},{ }^{24}$ then Eq. (6) is reduced to

$$
F_{P}=\frac{1+\alpha^{2}}{(1+\alpha)^{2}} \text {. }
$$

In the antiparallel (AP) configuration, we keep the definitions (5), characterizing each of the junctions as the relevant resistance ratios in the $\mathrm{P}$ configuration. Thus, assuming that the magnetization of the right electrode $(i=2)$ is reversed, one should substitute in Eq. (4) $R_{2 \uparrow} / R_{1 \uparrow}=\alpha \beta_{2}, R_{1 \downarrow} / R_{1 \uparrow}=\beta_{1}$, and $R_{2 \downarrow} / R_{2 \uparrow}=1 / \beta_{2}$. The corresponding Fano factor is then given by the formula

$$
F_{\mathrm{AP}}=\frac{\left(1+\alpha^{2} \beta_{2}^{2}\right)\left(\alpha+\beta_{1}\right)^{3}+\left(\alpha^{2}+\beta_{1}^{2}\right)\left(1+\alpha \beta_{2}\right)^{3}}{\left(1+\alpha \beta_{2}\right)^{2}\left(\alpha+\beta_{1}\right)^{2}(1+\alpha)\left(1+\beta_{1}\right)} .
$$

In the case of similar junctions with $\beta_{1}=\beta_{2}=\beta$, Eq. (8) reduces to

$$
F_{\mathrm{AP}}=\frac{\left(1+\alpha^{2} \beta^{2}\right)(\alpha+\beta)^{3}+\left(\alpha^{2}+\beta^{2}\right)(1+\alpha \beta)^{3}}{(1+\alpha \beta)^{2}(\alpha+\beta)^{2}(1+\alpha)(1+\beta)} .
$$

Let us introduce now the spin polarization $p_{i}$ as $p_{i}=\left(R_{i \downarrow}-\right.$ $\left.R_{i \uparrow}\right) /\left(R_{i \downarrow}+R_{i \uparrow}\right)$. When $\beta_{1}=\beta_{2}=\beta$, then also $p_{1}=p_{2}=$ $p$. The parameter $\beta$ is then related to the parameter $p$ via the formula $\beta=(1+p) /(1-p)$. The Fano factor (9) can then be rewritten as

$$
\begin{aligned}
F_{\mathrm{AP}}= & \frac{1}{2} \frac{\left[(1-p)^{2}+\alpha^{2}(1+p)^{2}\right][(1+p)+\alpha(1-p)]}{(1+\alpha)[1-p+\alpha(1+p)]^{2}} \\
& +\frac{1}{2} \frac{\left[(1+p)^{2}+\alpha^{2}(1-p)^{2}\right][(1-p)+\alpha(1+p)]}{(1+\alpha)[1+p+\alpha(1-p)]^{2}} .
\end{aligned}
$$

If apart from equal spin-filtering parameters, the system is additionally symmetric, i.e., $\alpha=1$, then $F_{P}=1 / 2$ in the parallel configuration [see Eq. (7)] and

$$
F_{\text {AP }}=\frac{1}{2}\left(1+p^{2}\right)
$$

in the antiparallel state [as follows from Eq. (10)]. This is in agreement with the calculations of Tserkovnyak et al. ${ }^{7}$

\section{B. All-magnetic structures}

Consider now a double-barrier junction with the central layer and the external electrodes being ferromagnetic. We keep the same parameters as before to describe the system. These parameters are defined by Eq. (5), in which all of the resistances are taken in the state with all magnetizations parallel (P state). The Fano factor in the $\mathrm{P}$ configuration can then be easily calculated, and we find it is given by Eq. (6).

The configuration with the magnetic moment of the central layer antiparallel to the magnetic moments of the outer ferromagnetic electrodes will be referred to in the following as the AP1 configuration, ( $\uparrow \downarrow \uparrow)$. In such a configuration, the corresponding resistance $R_{i \sigma}$ (for $i=1,2$ ) can be expressed by the resistances in the $\mathrm{P}$ configuration as $\sqrt{R_{i \uparrow} R_{i \downarrow}}$. In the limit of amorphous barriers, this approximation can be easily justified in terms of the densities of states (Julliere's model), but the applicability range of this approximation is broader. It is also a reliable approximation for epitaxial barriers since the resistance can be expressed as proportional to the product of average transmission probabilities through the two interfaces. Taking this into account, one obtains the corresponding Fano factor in the form

$$
F_{\mathrm{AP} 1}=\frac{\beta_{1}+\beta_{2} \alpha^{2}}{\left(\sqrt{\beta_{1}}+\sqrt{\beta_{2}} \alpha\right)^{2}} .
$$

In turn, in the configuration with one outer layer magnetized opposite to the other two layers [referred to as the AP2 configuration, $(\downarrow \uparrow \uparrow)]$, the resistances $R_{1 \sigma}$ of the left junction are given by the corresponding resistances in the parallel configuration as $\sqrt{R_{1 \uparrow} R_{1 \downarrow}}$. The corresponding Fano factor is then equal,

$$
F_{\mathrm{AP} 2}=\frac{\left(\beta_{1}+\alpha^{2}\right)\left(\sqrt{\beta_{1}}+\beta_{2} \alpha\right)^{3}+\left(\beta_{1}+\beta_{2}^{2} \alpha^{2}\right)\left(\sqrt{\beta_{1}}+\alpha\right)^{3}}{\left(\sqrt{\beta_{1}}+\alpha\right)^{2}\left(\sqrt{\beta_{1}}+\beta_{2} \alpha\right)^{2}\left(2 \sqrt{\beta_{1}}+\alpha+\beta_{2} \alpha\right)} .
$$




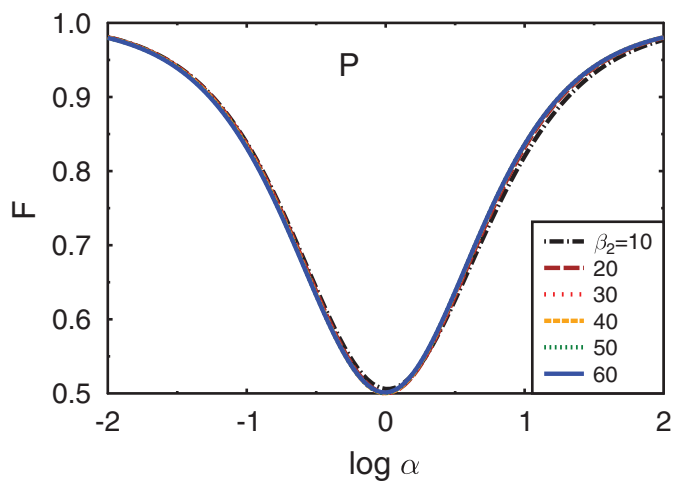

FIG. 1. (Color online) Fano factor in the $\mathrm{P}$ configuration for $\beta_{1}=$ 30 and different $\beta_{2}$ in the absence of spin relaxation.

Note that the Fano factor corresponding to the second AP2 configuration, $(\uparrow \uparrow \downarrow)$, can be obtained from the above formula by symmetry arguments. More specifically, the corresponding Fano factor can be obtained from Eq. (13) by the following replacements: $\beta_{1} \rightarrow \beta_{2}, \beta_{2} \rightarrow \beta_{1}$, and $\alpha \rightarrow 1 / \alpha$.

In a symmetric case with $\alpha=1$ and $\beta_{1}=\beta_{2}=\beta$, the above results reduce to

$$
F_{P, \mathrm{AP} 1}=1 / 2
$$

in the $\mathrm{P}$ and $\mathrm{AP} 1$ configurations, and

$$
F_{\mathrm{AP} 2}=\frac{1+\beta}{(1+\sqrt{\beta})^{2}}
$$

in the AP2 configuration.

The characteristic dependence of the Fano factor on the basic parameters of the model is presented in Figs. 1-3 for the P, AP1, and AP2 configurations, respectively. From Fig. 1 follows that the Fano factor in the P state is only weakly sensitive to the difference in the parameters $\beta$ of the two barriers, i.e., on the spin-filtering properties of the barriers. Apart from this, the Fano factor is minimum, $F \simeq 1 / 2$, in an almost symmetric situation $(\alpha \simeq 1)$. When the asymmetry increases, the Fano factor changes and tends to $F=1$ for highly asymmetric junctions.

The situation is different in the AP1 state (see Fig. 2), where the Fano factor is strongly sensitive to the difference in spin-filtering parameters of the two barriers. This is because the asymmetry effectively changes with the difference in the

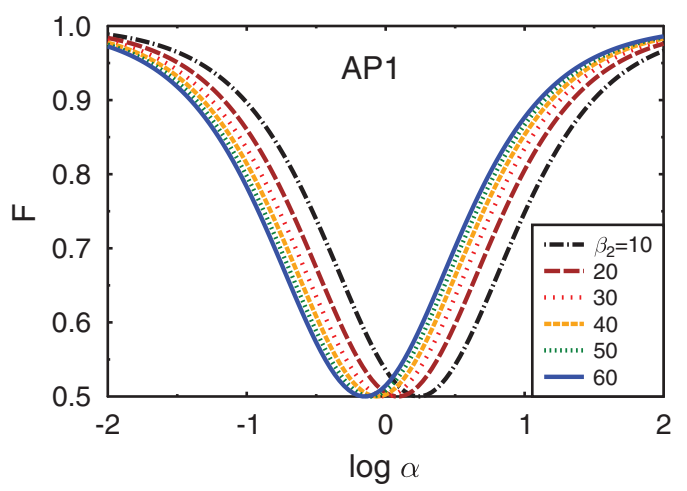

FIG. 2. (Color online) Fano factor in the AP1 configuration for $\beta_{1}=30$ and different $\beta_{2}$ in the absence of spin relaxation.

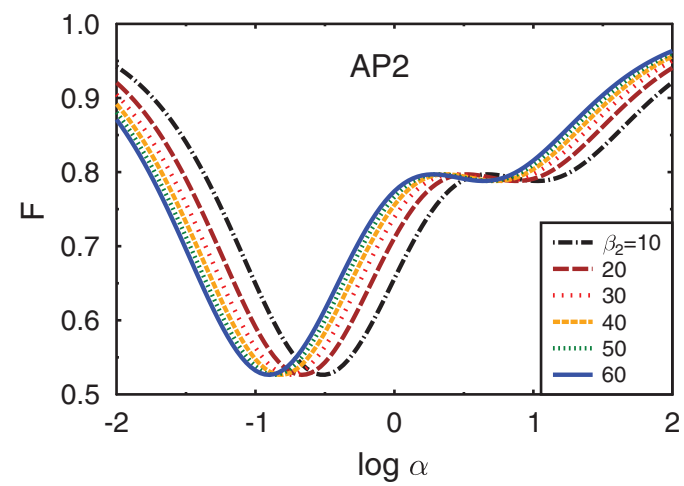

FIG. 3. (Color online) Fano factor in the AP2 configuration for $\beta_{1}=30$ and different $\beta_{2}$ in the absence of spin relaxation.

parameters $\beta$, and this in turn results in a shift in the magnitude of $\alpha$ at which the Fano factor is minimum. The minimum value of the Fano factor is still $F=1 / 2$. The Fano factor in the AP2 state is shown in Fig. 3. Its dependence on the parameter $\alpha$ is now qualitatively different. First, the minimum value of $F$ is larger than 0.5. Second, the point where the Fano factor has its minimum is shifted away from $\alpha=1$. Third, there is an additional weak local minimum in the dependence of the Fano factor on $\alpha$.

\section{SHOT NOISE IN THE PRESENCE OF SPIN RELAXATION}

Now we consider an all-magnetic structure and take into account spin relaxation in the central ferromagnetic layer. Spin relaxation affects the magnitude of spin fluctuations, while the charge fluctuations are not influenced by the spin-flip processes. The spin- $\sigma$ polarized current through the first (left) and second (right) tunnel barriers is given by the following formulas:

$$
\begin{aligned}
& I_{1 \sigma}=G_{1 \sigma}\left(\mu_{L}-\mu_{\sigma}\right) / e, \\
& I_{2 \sigma}=G_{2 \sigma}\left(\mu_{\sigma}-\mu_{R}\right) / e,
\end{aligned}
$$

where $G_{i \sigma}$ is the conductance of the $i$ th junction in the $\sigma$ spin channel, $\mu_{L}$ and $\mu_{R}$ are the chemical potentials in the left and right electrodes, respectively, while $\mu_{\sigma}$ is the spindependent chemical potential in the central layer. Here we assume that the electrons in the right and left electrodes are in quasiequilibrium, whereas $\mu_{\sigma}$ can fluctuate according to the fluctuating number of spin- $\sigma$ electrons in the central layer. The total current through the $i$ th barrier is the sum of partial currents in the spin channels, $I_{i}=I_{i \uparrow}+I_{i \downarrow}$.

The current fluctuations in the spin- $\sigma$ channel, $\Delta I_{i \sigma}$, consist of the contribution due to Langevin noise, $\delta I_{i \sigma}$, and due to fluctuations in the number of spin- $\sigma$ electrons in the central layer,

$$
\Delta I_{i \sigma}=\delta I_{i \sigma}+\frac{\partial I_{i}}{\partial \mu_{\sigma}} \delta \mu_{\sigma}
$$

In the following, we assume a short dwell time $\tau_{d}$, which means that the charge current is conserved at each moment $t$, so that

$$
\Delta I_{1}=\Delta I_{2},
$$


where $\Delta I_{i}$ is the current fluctuation in the $i$ th junction. In other words, the time scale associated with the noise of frequency $\omega$ should be longer than the dwell time, $\omega \tau_{d} \ll 1$. The dwell time for a symmetric system is given by the formula

$$
\frac{1}{\tau_{d}}=\frac{h}{2 e^{2}} \frac{v_{F}}{d} \frac{1}{R N},
$$

where $v_{F}$ is the Fermi velocity, $R$ is the barrier resistance, and $N$ is the number of transport channels, $N=k_{F}^{2} \Omega / 4 \pi$, with $k_{F}$ denoting the Fermi wave vector and $\Omega$ denoting the junction area. Taking parameters characterizing our junctions, we get $\tau_{d}$ of the order of $10^{-6} \mathrm{~s}$, which justifies our approximation for all magnetic configurations. Using Eqs. (16)-(18), we can rewrite the condition (19) in the following form:

$$
\begin{gathered}
\left(G_{1 \uparrow}+G_{2 \uparrow}\right) \delta \mu_{\uparrow}+\left(G_{1 \downarrow}+G_{2 \downarrow}\right) \delta \mu_{\downarrow} \\
=e\left(\delta I_{1 \uparrow}+\delta I_{1 \downarrow}-\delta I_{2 \uparrow}-\delta I_{2 \downarrow}\right) .
\end{gathered}
$$

We will describe the spin-relaxation process as a nonconservation of spin current transmitted through the central layer. Since the spin current through the $i$ junction is defined as $J_{i}=\left(I_{i \uparrow}-I_{i \downarrow}\right) / e$, one can write

$$
\Delta I_{1 \uparrow}-\Delta I_{1 \downarrow}-\Delta I_{2 \uparrow}+\Delta I_{2 \downarrow}=\frac{e \delta s}{\tau_{s}},
$$

where $\tau_{s}$ is the spin-relaxation time, $\delta s=\rho\left(\mu_{\uparrow}-\mu_{\downarrow}\right) \Omega d$ is the nonequilibrium variation of the total spin in the central layer of thickness $d$ and area $\Omega$, and $\rho$ is the electron density of states. Taking into account Eqs. (16)-(18), we can rewrite Eq. (22) as

$$
\begin{aligned}
& \left(G_{1 \uparrow}+G_{2 \uparrow}+g\right) \delta \mu_{\uparrow}-\left(G_{1 \downarrow}+G_{2 \downarrow}+g\right) \delta \mu_{\downarrow} \\
& =e\left(\delta I_{1 \uparrow}-\delta I_{1 \downarrow}-\delta I_{2 \uparrow}+\delta I_{2 \downarrow}\right),
\end{aligned}
$$

where we introduced the parameter $g$ defined as $g=$ $e^{2} \rho \Omega d / \tau_{s}$. Upon calculating $\delta \mu_{\uparrow, \downarrow}$ from Eqs. (21) and (23), and substituting them into Eq. (18), one arrives at the following relation between the total current fluctuation and its Langevin part:

$$
\begin{aligned}
\Delta I_{1}= & \delta I_{1 \uparrow}\left[1+\frac{G_{1 \uparrow}\left(2 G_{1 \downarrow}+2 G_{2 \downarrow}+g\right)+G_{1 \downarrow} g}{D}\right] \\
& +\delta I_{1 \downarrow}\left[1+\frac{G_{1 \downarrow}\left(2 G_{1 \uparrow}+2 G_{2 \uparrow}+g\right)+G_{1 \uparrow} g}{D}\right] \\
& -\delta I_{2 \uparrow} \frac{G_{1 \uparrow}\left(2 G_{1 \downarrow}+2 G_{2 \downarrow}+g\right)+G_{1 \downarrow} g}{D} \\
& -\delta I_{2 \downarrow} \frac{G_{1 \downarrow}\left(2 G_{1 \uparrow}+2 G_{2 \uparrow}+g\right)+G_{1 \uparrow} g}{D},
\end{aligned}
$$

where $D$ is defined as

$$
\begin{aligned}
D= & -\left(G_{1 \uparrow}+G_{2 \uparrow}\right)\left(G_{1 \downarrow}+G_{2 \downarrow}+g\right) \\
& -\left(G_{1 \downarrow}+G_{2 \downarrow}\right)\left(G_{1 \uparrow}+G_{2 \uparrow}+g\right) .
\end{aligned}
$$

The shot noise results from averaging of the square of $\Delta I^{(1)}$. As a result, one obtains

$$
\begin{aligned}
S= & \overline{\left(\delta I_{1 \uparrow}\right)^{2}}\left[1+\frac{G_{1 \uparrow}\left(2 G_{1 \downarrow}+2 G_{2 \downarrow}+g\right)+G_{1 \downarrow} g}{D}\right]^{2} \\
& +\overline{\left(\delta I_{1 \downarrow}\right)^{2}}\left[1+\frac{G_{1 \downarrow}\left(2 G_{1 \uparrow}+2 G_{2 \uparrow}+g\right)+G_{1 \uparrow} g}{D}\right]^{2}
\end{aligned}
$$

$$
\begin{aligned}
& +\overline{\left(\delta I_{2 \uparrow}\right)^{2}} \frac{\left[G_{1 \uparrow}\left(2 G_{1 \downarrow}+2 G_{2 \downarrow}+g\right)+G_{1 \downarrow} g\right]^{2}}{D^{2}} \\
& +\overline{\left(\delta I_{2 \downarrow}\right)^{2}} \frac{\left[G_{1 \downarrow}\left(2 G_{1 \uparrow}+2 G_{2 \uparrow}+g\right)+G_{1 \uparrow} g\right]^{2}}{D^{2}},
\end{aligned}
$$

where, for the Langevin noise $\overline{\left(\delta I_{1,2 ; \uparrow, \downarrow}\right)^{2}}$, we should substitute

$$
\overline{\left(\delta I_{1 \uparrow, \downarrow}\right)^{2}}=\overline{\left(\delta I_{2 \uparrow, \downarrow}\right)^{2}}=\frac{2|e V|}{R_{1 \uparrow, \downarrow}+R_{2 \uparrow, \downarrow}} .
$$

The Fano factor is then determined to be

$$
F=\frac{R_{\uparrow} R_{\downarrow} S}{2|e V|\left(R_{\uparrow}+R_{\downarrow}\right)},
$$

where $R_{\sigma}=R_{1 \sigma}+R_{2 \sigma}$, and $S$ is given by Eq. (26).

In the absence of spin relaxation, the parameter $g$ vanishes, $g=0$. After simple algebra, one recovers then the previously found result given by Eq. (4). In numerical calculations based on Eqs. (26)-(28), it is convenient to use the dimensionless parameter $\bar{g}=d / v_{F} \tau_{s}$ to characterize the spin-relaxation rate.

\section{A. Strong spin relaxation}

In the limiting case of $g \rightarrow \infty$ (strong spin relaxation), the accumulated spin fluctuations vanish, $\delta s=0$, which corresponds to $\delta \mu_{\uparrow}=\delta \mu_{\downarrow}$. Then, one finds the following expression for the Fano factor:

$$
F=\frac{R_{2 \uparrow}^{2} R_{2 \downarrow}^{2}\left(R_{1 \uparrow}+R_{1 \downarrow}\right)^{2}+R_{1 \uparrow}^{2} R_{1 \downarrow}^{2}\left(R_{2 \uparrow}+R_{2 \downarrow}\right)^{2}}{\left[R_{1 \uparrow} R_{1 \downarrow}\left(R_{2 \uparrow}+R_{2 \downarrow}\right)+R_{2 \uparrow} R_{2 \downarrow}\left(R_{1 \uparrow}+R_{1 \downarrow}\right)\right]^{2}} .
$$

The above formula can be used to calculate the Fano factor in various magnetic configurations, similarly as in the preceding section. Thus, in the $\mathrm{P}$ configuration, we find

$$
F_{P}=\frac{\alpha^{2} \beta_{2}^{2}\left(1+\beta_{1}\right)^{2}+\beta_{1}^{2}\left(1+\beta_{2}\right)^{2}}{\left[\beta_{1}\left(1+\beta_{2}\right)+\alpha \beta_{2}\left(1+\beta_{1}\right)\right]^{2}} .
$$

For the AP1 configuration ( $\uparrow \uparrow \uparrow)$, in turn, one finds

$$
F_{\mathrm{AP} 1}=\frac{\alpha^{2} \beta_{1} \beta_{2}^{2}+\beta_{1}^{2} \beta_{2}}{\left(\beta_{1} \sqrt{\beta_{2}}+\alpha \beta_{2} \sqrt{\beta_{1}}\right)^{2}} .
$$

Finally, Eq. (29) leads to the following Fano factor in the AP2 configuration $(\downarrow \uparrow \uparrow)$ :

$$
F_{\mathrm{AP} 2}=\frac{4 \alpha^{2} \beta_{1} \beta_{2}^{2}+\beta_{1}^{2}\left(1+\beta_{2}\right)^{2}}{\left[\beta_{1}\left(1+\beta_{2}\right)+2 \alpha \beta_{2} \sqrt{\beta_{1}}\right]^{2}} .
$$

As before, the Fano factor corresponding to the second AP2 configuration, $(\uparrow \uparrow \downarrow)$, can be obtained from the above formula by the following replacements: $\beta_{1} \rightarrow \beta_{2}, \beta_{2} \rightarrow \beta_{1}$, and $\alpha \rightarrow$ $1 / \alpha$.

The dependence of the Fano factor on the junction parameters and magnetic configuration in the limit of strong spin relaxation is shown in Figs. 4-6. The results shown in Figs. 4 and 5 are qualitatively similar to the corresponding ones in the absence of spin-relaxation processes; see Figs. 1 and 2, respectively. In turn, the results presented in Fig. 6 differ qualitatively from those shown in Fig. 3. The key difference is the absence of the second shallow minimum in the dependence of the Fano factor on the asymmetry parameter $\alpha$. Moreover, the absolute minimum value of $F$ is now smaller than in Fig. 3 and is equal to $1 / 2$, as in the other two configurations. 


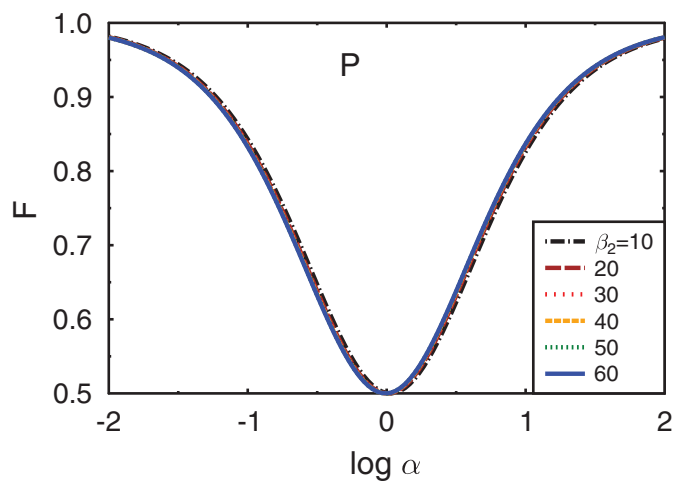

FIG. 4. (Color online) Fano factor in the P configuration for $\beta_{1}=$ 30 and $\beta_{2}$ as indicated in the limit of strong spin relaxation.

The above general formula in the limit of strong spin relaxation also applies to the case with a nonmagnetic central layer. In the case of parallel configuration, the Fano factor is then given by Eq. (30). Moreover, the formula (3) applies also to the antiparallel configuration. This is a consequence of the strong spin relaxation in the central layer, which breaks spin contact between the two ferromagnetic layers.

\section{TUNNEL MAGNETORESISTANCE OF THE DOUBLE-BARRIER STRUCTURE}

The model of shot noise described above contains several parameters characterizing the double-barrier junctions. These parameters can be evaluated from fitting to experimental observations. However, to get reliable values of these parameters from fitting, one should fit not only the shot noise, but also the resistance or tunnel magnetoresistance (TMR). Therefore, in this section, we briefly describe the TMR, which can be defined as the relative difference in the total junction resistance in the antiparallel and parallel states. Formally, we use the following definition:

$$
\mathrm{TMR}=\frac{I_{P}-I_{\mathrm{AP}}}{I_{\mathrm{AP}}}=\left(\frac{R_{\mathrm{AP}}}{R_{P}}-1\right),
$$

where $I_{P}\left(I_{\mathrm{AP}}\right)$ is the total current in the parallel (antiparallel) configuration, while $R_{P}\left(R_{\mathrm{AP}}\right)$ is the corresponding total resistance.

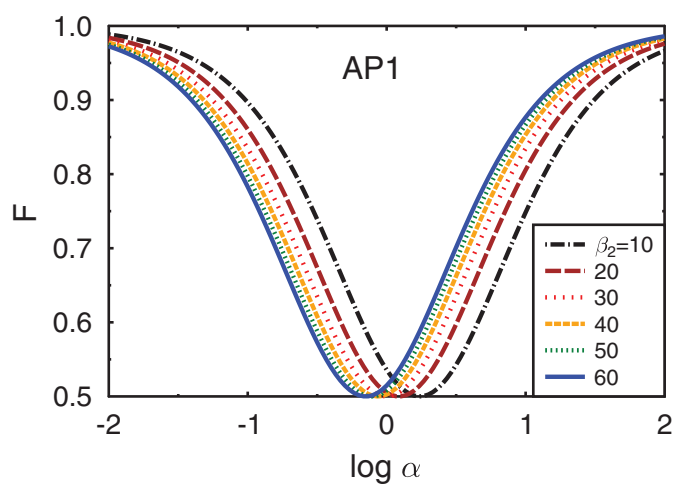

FIG. 5. (Color online) Fano factor in the AP1 configuration for $\beta_{1}=30$ and $\beta_{2}$ as indicated in the limit of strong spin relaxation.

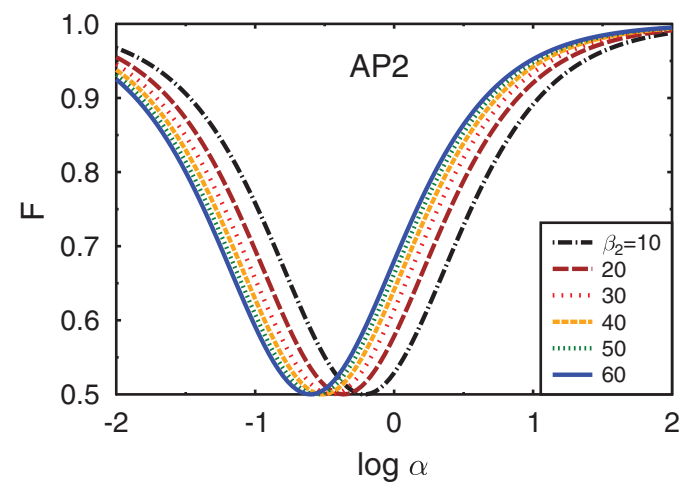

FIG. 6. (Color online) Fano factor in the AP2 configuration for $\beta_{1}=30$ and $\beta_{2}$ as indicated in the limit of strong spin relaxation.

The analysis of transport properties carried out in the preceding section allows one to determine the TMR in all magnetic states of the double-barrier junction. Thus, in the case of the AP1 configuration, we obtain

$$
\mathrm{TMR}_{\mathrm{AP} 1}=\frac{\left(\sqrt{\beta_{1}}+\alpha \sqrt{\beta_{2}}\right)\left(1+\alpha+\beta_{1}+\alpha \beta_{2}\right)}{2(1+\alpha)\left(\beta_{1}+\alpha \beta_{2}\right)}-1 .
$$

In turn, the TMR corresponding to the AP2 configuration is given by the formula

$$
\begin{aligned}
\mathrm{TMR}_{\mathrm{AP} 2}= & -1+\frac{1}{(1+\alpha)\left(\beta_{1}+\alpha \beta_{2}\right)}\left\{\left(\sqrt{\beta_{1}}+\alpha\right)\left(\sqrt{\beta_{1}}+\alpha \beta_{2}\right)\right. \\
& \left.\times\left(1+\alpha+\beta_{1}+\alpha \beta_{2}\right)\right\} .
\end{aligned}
$$

Variation of the TMR in the AP1 configuration with the asymmetry parameter $\alpha$ is shown in Fig. 7 for different values of the barrier spin-filtering parameters. Similar behavior can be expected for the second antiparallel configuration, AP2. By numerically fitting both the TMR and shot noise, one can more precisely determine the parameters used to describe the structure. To have the most reliable junction parameters, one should fit the TMR in all magnetic configurations.

\section{EXPERIMENTAL RESULTS}

The theoretical results have been compared with the experimental data obtained on $\mathrm{Fe} / \mathrm{MgO} / \mathrm{Fe} / \mathrm{MgO} / \mathrm{Fe}$ doublebarrier magnetic tunnel junctions. ${ }^{21}$ The junctions' growth, characterization, and the experimental techniques have

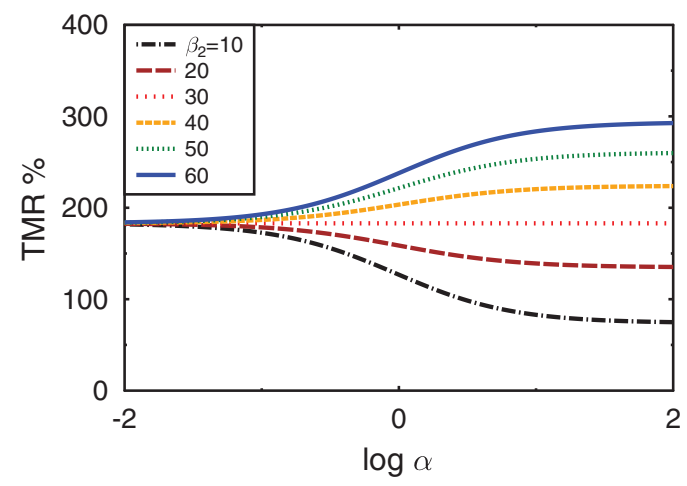

FIG. 7. (Color online) TMR as a function of $\alpha$ for $\beta_{1}=30$ and different values of $\beta_{2}$. 


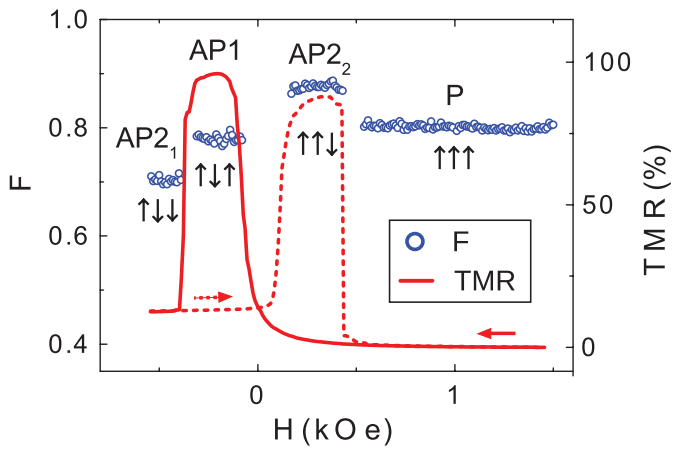

FIG. 8. (Color online) Shot noise and tunnel magnetoresistance at a fixed current during a magnetic field sweep at $T=0.3 \mathrm{~K}$.

already been described elsewhere. ${ }^{21-23}$ Shot noise and TMR were studied in $20 \times 20 \mu \mathrm{m}^{2}$ double-barrier magnetic tunnel junctions (DMTJs). This ensures a uniform distribution of the tunneling current over the junction area. Qualitatively similar results were obtained for the junction area between $10 \times 10$ and $30 \times 30 \mu \mathrm{m}^{2}$.

In this paper, we go beyond the experimental data presented in Ref. 23 by demonstrating the control and theoretical understanding of the shot noise and tunnel magnetoresistance in all four different magnetic states of a double-barrier magnetic tunnel junction. These four magnetic states are the parallel $(\mathrm{P})$ configuration ( $\uparrow \uparrow)$, antiparallel configuration with magnetic moment of the central layer opposite to the moments of the external electrodes $(\uparrow \downarrow \uparrow)$, denoted as AP1 configuration, and two AP2 configurations, $(\downarrow \uparrow \uparrow)$ and ( $\uparrow \downarrow)$, referred to in the following as $\mathrm{AP} 22_{1}$ and $\mathrm{AP} 22_{2}$ magnetic states.

As an example, Fig. 8 shows the dependence of the resistance and shot noise on the magnetic state of a sample described by $\alpha<1$. The measurements have been performed at a constant current, corresponding to $V=200 \mathrm{mV}$ in the $\mathrm{P}$ state. The sample had the following structure (numbers in brackets represent thickness in $\mathrm{nm}): \mathrm{MgO} / / \mathrm{MgO}(10) / \mathrm{Cr}(42) / \mathrm{Co}(10) / \mathrm{Fe} 1(5) / \mathrm{MgO}(3)$ $/ \mathrm{Fe} 2(5) / \mathrm{MgO}(2.7) / \mathrm{Fe} 3(10) / \mathrm{Co}(30) / \mathrm{Au}(10)$. Note that the barriers had slightly different thicknesses. Four distinct values of the resistance and shot noise have been observed, which correspond to the different magnetic configurations described above. Shot noise was measured over the voltage range $\pm 1 \mathrm{~V}$, and the average Fano values for each magnetic state were estimated from the bias range between 0.2 and $0.5 \mathrm{~V}$, since the influence of quantum well states (QWSs) was observed above $0.5 \mathrm{~V}$ at low temperatures (see Ref. 23).

The fitting of the experimental data to the theoretical results has been done numerically by finding the $\alpha$ and $g$ parameters, which give a set of $\left(\beta_{1}, \beta_{2}\right)$ parameters where the theoretical Fano factor agrees with the experimental data (with a tolerance of around $3-4 \%$ ) for all four states. In order to choose just one pair of $\left(\beta_{1}, \beta_{2}\right)$ values, we fitted the theoretical and experimental TMR values. From this, we found all of the parameters needed to describe the sample.

Figure 9 shows the fit of the theory to the shot-noise measurements in all four states for the sample from Fig. 8. The measurements were done at $T=60 \mathrm{~K}$, since the influence of QWSs gradually vanishes at high temperatures. It can be seen that the theory predicts quite well the Fano values for

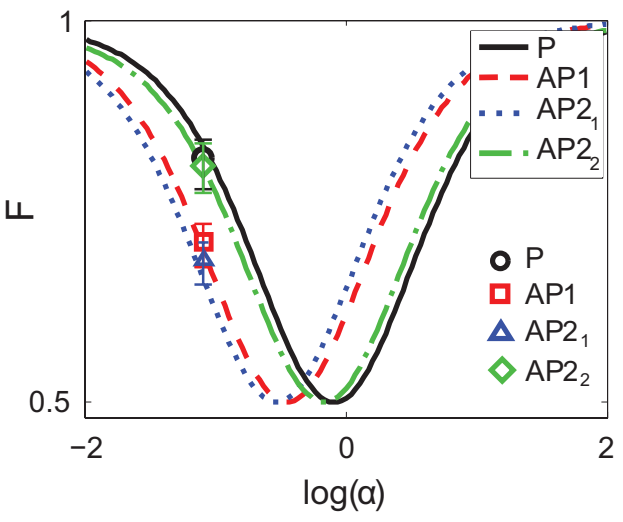

FIG. 9. (Color online) Comparison of the theory (lines) and experimental results (points) measured at $T=60 \mathrm{~K}$, for $\bar{g}=100$, $\alpha=0.08, \beta_{1}=3.5$, and $\beta_{2}=28.3$.

the parameters $\bar{g}=100, \alpha=0.08, \beta_{1}=3.5$, and $\beta_{2}=28.3$. These fitting parameters are in agreement with the observed properties of the junction. The parameter $\alpha=0.08$ comes from different thicknesses of the barriers (and also their different spin-filtering properties). On the other hand, $\beta_{1}=3.5$ and $\beta_{2}=28.3$ also make sense since $\beta_{1}$ corresponds to the thinner barrier, and generally thinner barriers have weaker spin-filtering properties than thicker ones. Finally, we note that the spin-relaxation rate parameter, $\bar{g}=d / v_{F} \tau_{s}$, with $d=5$ $\mathrm{nm}$ being the thickness of the central layer and the Fermi velocity $v_{F}$ of $10^{5} \mathrm{~m} / \mathrm{s}$, indicates a short spin-relaxation time $\tau_{s} \simeq 10^{-13} \mathrm{~s}$, which could be correlated with the fact that epitaxial $\mathrm{MgO}$ barriers of these thicknesses have a relatively high density of oblique defects. ${ }^{23}$

\section{SUMMARY}

We have analyzed the shot noise in double-barrier magnetic tunnel junctions. The central metallic layer of the junctions was either nonmagnetic or ferromagnetic. We have derived some simple analytical formulas for the Fano factor in different collinear magnetic configurations of the system in the limit of strong spin relaxation, as well as in the absence of spin relaxation. The tunnel junctions have been described by a couple of parameters, especially by the asymmetry parameter taking into account the difference in the two barriers, and also by two spin-filtering parameters (one for each barrier). These parameters have been evaluated from fitting to experimental data on tunnel magnetoresistance and shot noise (Fano factor).

The experimental data show that the Fano factor can be controlled by magnetic configuration of the system, and can take four different values corresponding to four different magnetic states. Theoretical evaluation of these factors agrees rather well with the experimental observations.

\section{ACKNOWLEDGMENTS}

We acknowledge M. Hehn, C. Tiusan, and A. Duluard for the preparation of samples. We also thank D. Herranz for his help with the experiment. This work is supported by the National Science Center in Poland as a research project in the years 2011-2014 and by the Polish National 
Center of Research and Development within the framework of the European project Era.Net.Rus "SpinBarrier" for the years 2012-2014. The work in Madrid has been supported by the Spanish MINECO (Grant No. MAT2012-32743, CONSOLIDER CSD2007-00010) and Comunidad de Madrid (Grant No. P2009/MAT-1726).
*Also at Faculty of Physics, Adam Mickiewicz University, Umultowska 85, 61-614 Poznań, Poland.

${ }^{1}$ Ya. M. Blanter and M. Büttiker, Phys. Rep. 336, 1 (2000).

${ }^{2}$ G. B. Lesovik, Pis'ma Zh. Eksp. Teor. 49, 513 (1989) [JETP Lett. 49, 592 (1989)].

${ }^{3}$ M. Büttiker, Phys. Rev. Lett. 65, 2901 (1990).

${ }^{4}$ C. W. J. Beenakker and M. Büttiker, Phys. Rev. B 46, 1889 (1992).

${ }^{5}$ B. R. Bulka, J. Martinek, G. Michalek, and J. Barnaś, Phys. Rev. B 60, 12246 (1999).

${ }^{6}$ B. R. Bulka, Phys. Rev. B 62, 1186 (2000).

${ }^{7}$ Y. Tserkovnyak and A. Brataas, Phys. Rev. B 64, 214402 (2001).

${ }^{8}$ R. Lopez and D. Sanchez, Phys. Rev. Lett. 90, 116602 (2003).

${ }^{9}$ A. Cottet, W. Belzig, and C. Bruder, Phys. Rev. Lett. 92, 206801 (2004).

${ }^{10}$ H. Birk, M. J. M. de Jong, and C. Schonenberger, Phys. Rev. Lett. 75, 1610 (1995).

${ }^{11}$ F. M. Souza, A. P. Jauho, and J. C. Egues, Phys. Rev. B 78, 155303 (2008).

${ }^{12}$ A. L. Chudnovskiy, J. Swiebodzinski, and A. Kamenev, Phys. Rev. Lett. 101, 066601 (2008).

${ }^{13}$ Y. Yamauchi, K. Sekiguchi, K. Chida, T. Arakawa, S. Nakamura, K. Kobayashi, T. Ono, T. Fujii, and R. Sakano, Phys. Rev. Lett. 106, 176601 (2011).
${ }^{14}$ I. Zutić, J. Fabian, and S. Das Sarman, Rev. Mod. Phys. 76, 323 (2004).

${ }^{15}$ A. Gokce, R. Stearrett, E. R. Nowak, and C. Nordman, Fluctuation Noise Lett. 10, 381 (2011).

${ }^{16}$ R. Guerrero, F. G. Aliev, Y. Tserkovnyak, T. S. Santos, and J. S. Moodera, Phys. Rev. Lett. 97, 266602 (2006).

${ }^{17}$ R. Guerrero, D. Herranz, F. G. Aliev, F. Greullet, C. Tiusan, M. Hehn, and F. Montaigne, Appl. Phys. Lett. 91, 132504 (2007).

${ }^{18}$ T. Arakawa, K. Sekiguchi, S. Nakamura, K. Chida, Y. Nishihara, D. Chiba, K. Kobayashi, A. Fukushima, S. Yuasa, and T. Ono, Appl. Phys. Lett. 98, 202103 (2011).

${ }^{19}$ T. Arakawa, T. Tanaka, K. Chida, S. Matsuo, Y. Nishihara, D. Chiba, K. Kobayashi, T. Ono, A. Fukushima, and S. Yuasa, Phys. Rev. B 86, 224423 (2012).

${ }^{20}$ K. Liu, K. Xia, and G. E. W. Bauer, Phys. Rev. B 86, 020408R (2012).

${ }^{21}$ C. Tiusan, F. Greullet, M. Hehn, F. Montaigne, S. Andrieu, and A. Schuhl, J. Phys.: Condens. Matter 19, 165201 (2007).

${ }^{22}$ D. Herranz, F. G. Aliev, C. Tiusan, M. Hehn, V. K. Dugaev, and J. Barnaś, Phys. Rev. Lett. 105, 047207 (2010).

${ }^{23}$ J. P. Cascales, D. Herranz, F. G. Aliev, T. Szczepański, V. K. Dugaev, J. Barnaś, A. Duluard, M. Hehn, and C. Tiusan, Phys. Rev. Lett. 109, 066601 (2012).

${ }^{24}$ It should be noted that in reality the junctions can be described by different $\beta_{1}$ and $\beta_{2}$ even when the material is the same. 